\title{
Lello Savonardo: Sociologia della musica. La costruzione sociale del suono dalle tribù al digitale. Torino: UTET Università, 2010. ISBN 978-8860082473. 288 pagine.
}

Nell'ambito della riflessione sociologica sulla musica, il volume di Lello Savonardo Sociologia della musica. La costruzione sociale del suono dalle tribù al digitale rappresenta uno dei manuali di riferimento nel panorama della produzione scientifica italiana di settore. Nato da una lunga esperienza di studio e ricerca dell'autore al confine tra musica, sociologia e comunicazione, il testo si propone come guida introduttiva allo studio della sociologia della musica e offre al lettore una puntuale sistematizzazione delle teorie, dei temi e delle questioni più rilevanti che animano il dibattito intorno alla disciplina. L'autore analizza il complesso rapporto tra i linguaggi musicali e la realtà sociale, con costante riferimento alle trasformazioni introdotte dallo sviluppo dei media, restituendo un'analisi approfondita e ampiamente documentata delle origini, delle tappe evolutive e dello stato attuale della sociologia della musica. Nel 2015 è stata pubblicata l'edizione francese del testo, intitolata Sociologie de la musique. La construction sociale du son des 'tribus' au numérique.

Assumendo la musica come punto di osservazione privilegiato della realtà sociale, la tesi sostenuta da Savonardo è che grazie allo studio dei linguaggi musicali e delle dinamiche che caratterizzano la produzione, la distribuzione e la fruizione musicale è possibile leggere e comprendere in profondità le trasformazioni sociali del nostro tempo e i processi individuali e collettivi che contribuiscono a generarle. Con questo volume, infatti, l'autore si fa portavoce di una moderna sociologia della musica, in cui l'intreccio tra musica e mutamento sociale rappresenta il concetto guida.

Il lavoro di Lello Savonardo, come testimonia anche il titolo del volume, è ispirato profondamente dalla sociologia fenomenologica di Berger e Luckmann (1966): la musica e l'esperienza sonora nel suo complesso, infatti, sono concepite come parte di un processo di costruzione sociale dialetticamente strutturato, in cui convivono simultaneamente elementi creativi e condizionamenti strutturali, innovazione e tradizione, fattori individuali e collettivi. Secondo l'autore, la musica si manifesta in un dato contesto culturale allo stesso tempo come 'riflesso' e 'sintomo' dell'esperienza sociale, incarnando uno degli elementi simbolici costitutivi del processo di costruzione della realtà. Più precisamente, nell'introduzione al volume, l'autore chiarisce che

[1]a musica, così come ogni forma artistica e culturale, può esprimere una significativa carica creativa, ma anche una rilevante funzione di conservazione. [...] La musica, e più in generale l'arte, rappresenta un elemento rilevante nei processi di coesione sociale e 
di conservazione della memoria poiché tradizionalmente le società utilizzano la mediazione delle istituzioni artistiche e culturali per 'ricordare' il proprio passato. In tal senso, l'arte assume un ruolo particolarmente significativo nei processi di costruzione sociale della realtà. (XVII)

Facendo propria la visione dell'antropologo ed etnomusicologo Alan P. Merriam (1964), Savonardo attribuisce alla sociologia il compito di riconoscere il peso specifico della musica nella più ampia analisi del mutamento sociale e di osservarla sia in relazione al contesto culturale di riferimento: music in culture, sia come espressione stessa della cultura: music as culture.

Nella prefazione al volume, il sociologo Franco Crespi evidenzia i principali contributi che il libro offre alla comunità scientifica e pone l'accento, in primo luogo, proprio sull'originalità con cui l'autore mette a fuoco lo studio della musica in relazione alla dinamica sociale. Secondo Crespi, il testo di Savonardo ha anche l'indubbio merito di fornire legittimità alla sociologia della musica come specifico campo di studi, grazie all'accurata ricostruzione delle origini e delle evoluzioni della disciplina nel tempo e delle principali teorie sociologiche che hanno per oggetto i fenomeni musicali.

Un ulteriore aspetto che rende interessante il lavoro del sociologo partenopeo, come suggerisce il massmediologo Derrick de Kerckhove nella postfazione al volume, è lo sguardo costantemente rivolto alle relazioni tra i prodotti sonori, il ruolo della tecnologia e i conseguenti processi creativi nelle diverse epoche - "dalle tribù al digitale" - che rende il lavoro di Savonardo ancorato al passato, ma proiettato al futuro.

Inoltre, un glossario di 111 voci posto alla fine del testo costituisce un valido strumento di disambiguazione concettuale, sempre più necessaria nell'ambito di una disciplina relativamente giovane. Il volume, dunque, si configura come una vera e propria bussola di orientamento nel campo della sociologia della musica e propone un percorso di lettura ricco e articolato, strutturato in due parti.

Nella prima parte del testo, l'autore ricostruisce le origini della sociologia della musica attingendo al patrimonio di studi ereditato dai padri ispiratori e fondatori della disciplina, come Georg Simmel, Max Weber, Theodor W. Adorno e Alfred Schütz.

Attraverso il contributo di Georg Simmel, espresso nell'opera Studi psicologici ed etnologici sulla musica (Psychologische und ethnologische Studien über Musik) del 1881, Savonardo ricerca le origini della musica come fenomeno sociale, esplorando i processi musicali, il rapporto tra artista e fruitore, nonché le relazioni tra l'opera d'arte, l'individuo e la società. L'autore evidenzia nel primo capitolo come gli studi di Simmel sulla musica siano un primo tentativo di connettere la musica ad altri elementi legati alla dimensione più intima dell'individuo e alla realtà sociale.

Nel secondo capitolo, Savonardo ripercorre la nascita ufficiale della sociologia della musica, sancita dal saggio di Max Weber I fondamenti razionali e sociologici della musica (Die rationalen und soziologischen Grundlagen der Musik), pubblicato postumo per la prima volta nel 1921. Passando in rassegna le teorie del sociologo tedesco, il testo analizza la significativa interdipendenza tra le forme musicali e la realtà sociale nell'ambito del crescente processo 
di razionalizzazione che caratterizza la modernità occidentale. L'autore opera, inoltre, un parallelismo tra le teorie di Simmel e Weber per individuare alcuni elementi comuni nella riflessione sull'evoluzione della musica. Secondo Savonardo, per entrambi i sociologi la musica nasce prima come esigenza espressiva, ma nel corso del tempo i linguaggi musicali si oggettivano e acquisiscono una propria autonomia, fino a decretare il mutamento del ruolo della musica nella società, che passa da una funzione tipicamente espressiva a una vera e propria forma d'arte.

La relazione tra arte e società è uno dei temi cruciali del libro ed è affrontato in modo particolare nel terzo capitolo dedicato al pensiero talvolta contraddittorio di Theodor W. Adorno, esponente della teoria critica della Scuola di Francoforte. Savonardo mette bene in evidenza che il principale obiettivo della sociologia della musica, nell'ottica adorniana, è lo studio delle relazioni esistenti tra i fenomeni musicali, gli ascoltatori, l'ideologia e le classi sociali (Adorno 1962). Nei suoi numerosi saggi dedicati al tema, infatti, Adorno sostiene che le forme musicali riflettono le ideologie e le forme di repressione che caratterizzano la società di massa di tipo capitalistico. Savonardo chiarisce tuttavia che, secondo Adorno, la musica non è soltanto un prodotto dell'ideologia dominante, ma può essere interpretata anche come forma di opposizione e critica al sistema sociale stesso.

Mentre Adorno adotta un approccio macro-sociologico, cioè rivolto alla comprensione dei grandi processi storico-sociali e dei comportamenti di massa, nel quarto capitolo Savonardo analizza anche gli aspetti micro-sociologici dedicando un approfondimento al pensiero di Alfred Schütz, espresso nell'opera La fenomenologia del mondo sociale (Der sinnhafte Aufban der sozialen Welt) del 1932, e al suo approccio fenomenologico alla musica, a partire dai lavori Frammenti di fenomenologia della musica (Fragments on the Phenomenology of Music), scritto nel 1944 e pubblicato postumo nel 1976, e Fare musica insieme. Studio sulla relazione sociale (Making Music Together. A Study in Relationship) del 1951. Il filosofo austriaco, restringendo il campo d'indagine all'intersoggettività, studia l'interazione tra il compositore, l'esecutore e l'audience, ma anche la "comunicazione" e il "significato" dell'opera musicale, socialmente costruiti. Attraverso l'analisi delle teorie di Schütz, Savonardo si sofferma al tempo stesso sul carattere sociale e non-sociale della musica, facendo emergere i processi comunicativi che la caratterizzano all'interno di una struttura sociale in cui interagiscono compositore, esecutore ed ascoltatore.

A partire dal quadro teorico delineato nella prima parte del volume, nella seconda parte il focus si sposta sulle principali trasformazioni che, a partire dal secondo dopoguerra, determinano mutamenti tecnologici, sociali e culturali particolarmente significativi in ambito musicale. L'autore, infatti, propone approfondimenti tematici e riflessioni teoriche che investono il rapporto tra musica, media e culture di massa, con un affondo sulla popular music, i mass media e le culture giovanili, fino ad arrivare agli snodi più recenti del dibattito che riguardano le connessioni tra musica, new media e culture digitali. Filo conduttore della seconda parte del volume è la relazione tra i diversi linguaggi musicali e lo sviluppo tecnologico dei media. Prendendo in prestito le parole dell'autore, 
[1]'evoluzione dei mezzi di comunicazione si intreccia strettamente con le principali trasformazioni, sociali, culturali ed istituzionali del mondo moderno. I significativi cambiamenti, la pluralità e la complessità delle forme culturali, insieme alla molteplicità dei contesti sociali pongono la sociologia della musica e dell'arte dinanzi alla necessità di utilizzare nuove categorie interpretative che permettano di leggere tali mutamenti. I prodotti artistici sono inseriti all'interno di dinamiche economiche e di potere che orientano i processi culturali e ne sono a loro volta influenzate, in una relazione di reciprocità. (XXIV)

La seconda parte del volume si apre con un capitolo incentrato su alcune delle principali teorie dei sociologi Howard S. Becker e Pierre Bourdieu, che offrono all'autore spunti di riflessione e categorie interpretative utili per argomentare le molteplici relazioni che intercorrono tra i sistemi sociali e le forme di produzione e fruizione culturale, artistica e musicale che in essi si realizzano.

In particolare, il contributo più rilevante di Becker (1982), esponente della Nuova Scuola di Chicago, riguarda la sua teoria sui mondi dell'arte (art worlds) o mondi culturali. Attraverso il lavoro del sociologo americano, Savonardo mostra come l'arte possa avere allo stesso tempo un carattere integrato nel sociale e una forza innovativa che si contrappone all'ordine costituito. Inoltre, viene messa in evidenza l'idea di Becker secondo la quale la produzione artistica non è appannaggio dell'artista, ma è il risultato di un processo collettivo nel quale, oltre all'artista, gioca un ruolo fondamentale il personale di supporto.

Diverse sono le categorie concettuali che Savonardo riprende anche dal pensiero di Pierre Bourdieu per arricchire la propria riflessione sul rapporto tra arte, musica e società. L'autore mostra come nella società di massa l'opera d'arte sia il risultato di un processo collettivo "strutturato" e "strutturante", in cui gli elementi legati alla personalità individuale sono legati al capitale sociale, culturale ed economico posseduto e a diversi altri fattori. Secondo Bourdieu, infatti, i fenomeni artistici e musicali si collocano in un dato contesto storico, sociale e culturale e si realizzano all'interno delle dinamiche di interazione tra diversi campi.

Uno degli aspetti maggiormente sottolineati da Savonardo in riferimento alle teorie di Bourdieu riguarda il concetto di "gusto" in ambito artistico e musicale (Bourdieu 1979). Le preferenze di gusto, secondo Bourdieu, non sono espressione soltanto di particolari predisposizioni individuali, ma sono influenzate e in alcuni casi determinate anche dall'appartenenza sociale. Con l'avvento e lo sviluppo delle culture di massa, sottolinea Savonardo, la relazione tra gusto artistico e appartenenza sociale diventa sempre meno lineare e la distinzione tra arte d'élite e arte di massa si declina lungo dimensioni inedite, che includono nuove forme di ibridazione culturale e nuove pratiche di consumo e di riproduzione delle diseguaglianze sociali.

Tali osservazioni risultano particolarmente interessanti rispetto all'approfondimento che l'autore propone nel sesto capitolo, incentrato sulla popular music e le culture di massa. È qui, infatti, che si può intercettare uno dei contributi più importanti del lavoro di Savonardo, ovvero una disanima critica e ampiamente documentata delle numerose defini- 
zioni di popular music presenti in letteratura. Sposando la posizione di Richard Middleton (1990), Savonardo critica la frammentarietà delle definizioni più comuni sostenendo che è necessario considerare le forme di popular music storicamente individuate all'interno dell'intero campo storico-musicale, tenendo sempre conto delle continue trasformazioni in corso. In particolare, Savonardo mette in risalto la stretta connessione tra la popular music, i media, le culture di massa, l'universo giovanile e i suoi linguaggi espressivi, temi che l'autore tratta in modo approfondito nel volume Pop music, media e culture giovanili. Dalla Beat Revolution alla Bit Generation (2017). Andando più a fondo, l'autore sostiene che

[1]'industria culturale e i mass media svolgono un ruolo sempre più determinante nei processi di produzione e di fruizione musicale, influenzando le forme di ascolto e di consumo giovanile, e non solo. Tuttavia, i mass media, da un lato, tendono a determinare l'omologazione dei consumi culturali e l'appiattimento dei gusti del pubblico e, dall'altro, consentono alla musica pop, anche quando propone nuove tendenze e linguaggi, di svilupparsi e consolidarsi, raggiungendo un pubblico sempre più vasto. (XXVI)

Lo sviluppo dei media e delle tecnologie di riproduzione e fruizione musicale sono al centro della riflessione di Savonardo negli ultimi due capitoli del volume. L'autore si concentra sulle principali trasformazioni che investono il rapporto tra musica e tecnologia, ripercorrendo l'evoluzione dei media dalla fase analogica a quella digitale ed evidenziando in particolare l'emergere di nuovi processi di ibridazione tra i diversi linguaggi musicali e nuove modalità creative.

Nel settimo capitolo, l'autore riprende le riflessioni di Walter Benjamin (1936) sulla riproducibilità tecnica dell'opera d'arte e discute delle implicazioni generate dallo sviluppo dei media, soprattutto in riferimento alle modalità di fruizione culturale di massa e al rapporto tra l'artista e la produzione musicale. Savonardo si interroga anche sulla crisi che vive l'opera d'arte nella società industriale e sull'idea stessa di originalità e autenticità. Attraverso le teorie di Marshall McLuhan (1964), Walter Ong (1982) e Derrick de Kerckhove (Buffardi 2004), l'autore sottolinea, inoltre, che i mass media, ed in particolare la radio e la televisione, introducono una "oralità secondaria" che riporta l'uomo nel mondo della sonorità, dell'emozione e della simultaneità temporale.

La nascita e lo sviluppo delle tecnologie digitali e dei new media, descritta nell'ottavo capitolo, diventa per Savonardo l'occasione per avviare una riflessione sulle potenzialità espressive e creative delle produzioni artistiche nell'era digitale e sulle conseguenti trasformazioni delle modalità di fruizione musicale. A differenza dei media analogici che moltiplicano i momenti di aggregazione e socializzazione collettiva, Savonardo sottolinea che i new media favoriscono una nuova dimensione di ascolto privato, nella sfera domestica e individuale, ma allo stesso tempo estendono l'interattività, la multimedialità e la connettività. L'autore, inoltre, esamina con grande pertinenza il passaggio dall'analogico al digitale e le modalità con cui i new media liberano la musica dal suo supporto, grazie all'avvento prima del cd e poi dell'mp3. 
Infine, il sociologo partenopeo indaga le trasformazioni che investono il processo creativo, il ruolo dell'artista e l'identità dell'opera d'arte nell'era digitale, giungendo a considerazioni di particolare interesse. Riprendendo le riflessioni di Pierre Lévy (1997) sull'opera d'arte nel contesto della cybercultura e parafrasando Walter Benjamin (1936), Savonardo definisce una nuova categoria interpretativa per chiarire la condizione contemporanea dell'opera d'arte, prodotta, distribuita e fruita "nell'epoca della sua irriproducibilità digitale":

Le riflessioni di Lévy sull'“opera d'arte", che potremmo, dunque, considerare nell'epoca della sua "irriproducibilità digitale", sembrano mettere parzialmente in crisi le teorie di Benjamin (1936). Infatti, da un lato tendono a capovolgere le riflessioni dello studioso tedesco sull'autenticità - poiché l'opera d'arte, nella cybercultura, ritorna ad essere considerata un oggetto unico e irripetibile che trae il suo valore dal suo essere hic et nunc; e dall'altro, sembrano estremizzare il concetto espresso da Benjamin sulla morte dell'autore. Con le tecnologie interattive, infatti, entra completamente in crisi il ruolo dell'autore che perde la paternità dell'opera nell'interazione con il fruitore, il quale diventa co-autore e contribuisce alla realizzazione dell'opera stessa, rendendola irripetibile nelle stesse modalità e nelle stesse forme che quella specifica interazione determina. Le nuove tecnologie digitali e il loro utilizzo sembrano, quindi, mettere in discussione e talvolta capovolgere le categorie estetiche e i canoni artistici tradizionali, ma anche le nostre modalità di interazione quotidiana. (197)

In conclusione, il testo di Lello Savonardo suggerisce diversi spunti di riflessione per lo studio delle trasformazioni socio-culturali che investono l'universo sonoro. L'autore, infatti, dedica le ultime pagine del suo lavoro al tema della costruzione sociale dell'esperienza sonora, esponendo la propria visione dei rapporti tra musica, connessioni, innovazioni, creatività e interattività. Il testo si chiude con l'individuazione di alcuni percorsi che potrebbero guidare la futura riflessione sociologica sul ruolo del suono e della musica nelle società contemporanee. Secondo Savonardo, una moderna sociologia della musica dovrebbe perseguire l'obiettivo di cogliere ed interpretare le relazioni tra i molteplici fattori che contribuiscono alla costruzione dell'immaginario sonoro, nei diversi contesti socio-culturali, allargando i confini disciplinari e gli ambiti di ricerca.

\section{Rosanna MARINO (Napoli)}

\section{Note}

1 Rosanna Marino, PhD in Scienze Sociali e Statistiche, svolge attività didattica integrativa nel settore della Sociologia dei processi culturali e comunicativi presso il Dipartimento di Scienze Sociali dell'Università degli Studi di Napoli Federico II. 


\section{Bibliografia}

Adorno, Theodor W.: Einleitung in die Musiksoziologie. Zwölf theorische Vorlesungen. Frankfurt am Main: Suhrkamp Verlag, 1962.

Becker, Howard S.: Art worlds. Berkeley: University of California Press, 1982.

Benjamin, Walter: Das Kunstwerk im Zeitalter seiner technischen Reproduzierbarkeit. Frankfurt: Suhrkamp Verlag, 1936.

Berger, Peter L. / Luckmann, Thomas: The Social Construction of Reality. Garden City New York: Doubleday and Co., 1966.

Bourdieu, Pierre: La distinction. Critique sociale du jugement. Paris: Éditions de Minuit, 1979.

Buffardi, Annalisa: "Il pensiero digitale e l'arte della connessione. Conversazione con Derrick de Kerckhove”. In Savonardo, Lello (ed.): Musicman_machine. Arte e nuove tecnologie nell'era digitale. Napoli: Graus, 2004, 97-119.

Lévy, Pierre: Cyberculture. Rapport au Conseil de l'Europe. Paris: Odile Jacob, 1997.

McLuhan, Marshall: Understanding Media. Cambridge: MIT Press, 1964.

Merriam, Alan P.: The Anthropology of Music. Evanstone: Northwestern University Press, 1964.

Middleton, Richard: Studying Popular Music. Buckingham: Open University Press, 1990.

Ong, Walter J.: Orality and Literacy. The Technologizing of the World. New York: Methuen, 1982.

Savonardo, Lello: Sociologie de la musique. La construction sociale du son des 'tribus' au numérique. Trad. Malika Combes et Amélie Munoz. Paris: L'Harmattan, 2015.

Savonardo, Lello: Pop music, media e culture giovanili. Dalla Beat Revolution alla Bit Generation. Milano: Egea, 2017.

Schütz, Alfred: Der sinnhafte Aufbau der sozialen Welt. Eine Einleitung in die verstehende Soziologie. Frankfurt am Main: Suhrkamp, 1932.

Schütz, Alfred: "Making music together. A study in relationship". In: Schütz, Alfred: Collected Papers. Vol. II: Studies in Social Theory. Ed. Arvid Brodersen. The Hague: Martinus Nijhoff, 1964, 159-178.

Schütz, Alfred: "Fragments on the Phenomenology of Music". In: Smith, F. Joseph (ed.): In Search of Musical Method. New York: Gordon \& Breach, 1976, 5-72.

Simmel, Georg: "Psychologische und ethnologische Studien über Musik”. In: Zeitschrift für Völkerpsychologie und Sprachwissenschaft 13,3 (1881), 261-305.

Weber, Max: Die rationalen und soziologischen Grundlagen der Musik. München: Drei Masken, 1921. 